\title{
Minimal muon anomalous magnetic moment
}

\author{
Carla Biggio $^{a}$ and Marzia Bordone ${ }^{b}$ \\ ${ }^{a}$ Dipartimento di Fisica, Università di Genova $\mathcal{G}$ INFN, Sezione di Genova, \\ via Dodecaneso 33, 16159 Genova, Italy \\ ${ }^{b}$ Physik-Institut, Universität Zürich, \\ Winterthurerstrasse 190, CH-8057 Zürich, Switzerland \\ E-mail: biggio@ge.infn.it, marzia.bordone@physik.uzh.ch
}

ABstract: We classify all possible one-particle (scalar and fermion) extensions of the Standard Model that can contribute to the anomalous magnetic moment of leptons. We review the cases already discussed in the literature and complete the picture by performing the calculation for a fermionic doublet with hypercharge $-3 / 2$. We conclude that, out of the listed possibilities, only two scalar leptoquarks and the pseudoscalar of a peculiar twoHiggs-doublet model could be the responsibles for the muon anomalous magnetic moment discrepancy. Were this the case, this particles could be seen in the next LHC run. To this aim, especially to test the leptoquark hypothesis, we suggest to look for final states with tops and muons.

Keywords: Beyond Standard Model, Electromagnetic Processes and Properties ARXiv EPRINT: 1411.6799 


\section{Contents}

1 Introduction 1

2 The classification $\quad 2$

2.1 Fermions 3

2.2 Scalars 4

3 The contribution of the colourless SU(2) doublet with hypercharge $-3 / 2 \quad 4$

4 Other cases $\quad 6$

4.1 Fermions 6

$\begin{array}{lll}4.2 & \text { Scalars } & 7\end{array}$

$\begin{array}{llr}5 & \text { Conclusions } & 9\end{array}$

$\begin{array}{ll}\text { A Lagrangian in the mass basis } & 10\end{array}$

\section{Introduction}

The anomalous magnetic moments of electron and muon are among the best measured quantities ever. With its 12 digits, the measurement of the anomalous magnetic moment of the electron is used to fix the value of the fine structure constant $\alpha_{e m}$, while the muon anomalous magnetic moment, $a_{\mu}=\frac{g-2}{2}$, can be used to test the Standard Model (SM) or, in other words, to search for new physics.

In 2006 the experiment E821 carried out at the Brookhaven laboratories measured [1]

$$
a_{\mu}^{\exp }=116592080(63) \cdot 10^{-11},
$$

while the value predicted within the SM is given by [2]

$$
a_{\mu}^{\mathrm{SM}}=116591790(65) \cdot 10^{-11} .
$$

The difference between the predicted and measured value, $\Delta a_{\mu}=290(90) \cdot 10^{-11}$, constitutes a discrepancy with $3.1 \sigma$ significance.

The SM result is not univocal, since the hadronic contribution depends on some experimental inputs. The above quoted result is obtained by taking data from $e^{+} e^{-}$annihilation. If, on the other hand, one uses $\tau$-decay data, a slightly higher value is obtained [3], leading to a smaller $\Delta a_{\mu}$. Therefore one could think that the reason of such a discrepancy is due to a poor knowledge of the theoretical calculation. However, if one fixes by hand the hadronic contribution in order to obtain the experimental result, other SM predictions turn out to 
be affected, in such a way that this possibility is substancially excluded [4-6]. Therefore, the reason of the discrepancy seems not to lie in the SM calculation.

On the other hand, the measurement of the $(g-2)_{\mu}$ has been performed only by one experiment, so that the possibility exists that some systematics out of control affect the result. In order to have a confirmation or a disproof of it, the experiment E989 is under construction at Fermilab [7] and another one has been proposed in Japan, at J-PARC [8].

Therefore, if one takes the experimental result as firm as well as the SM calculation, the only possibility to explain the discrepancy is to invoke the presence of new physics (NP). In the following we will make this assumption and analyse which kind of NP could be there.

The contribution of new physics to the muon anomalous magnetic moment has been considered in many extensions of the SM. For example, in supersymmetric (SUSY) models it is quite easy to get the needed $\Delta a_{\mu}$, when two sparticles circulate in the loop. However, this is not the only possibility and, in particular, it is possible to give a positive contribution to $a_{\mu}$ with the simple addition of one new particle. In this paper we focus precisely on this hypothesis and classify all possible one-particle SM extensions that can give rise, at one loop, to a contribution to the anomalous magnetic moment of the muon. We consider only scalars and fermions. Indeed the addition of new massive vector bosons would imply the extension of the SM gauge group, a possibility that we do not want to consider here. Most of the cases that we will find in our general classification have been already studied in the literature. Here we aim to complete the picture by calculating the contribution to $a_{\mu}$ given by the addition to the SM of a fermionic colourless $\mathrm{SU}(2)$ doublet with hypercharge $-3 / 2$, a result which is not present in the literature. Afterwards, we briefly review the literature regarding the other cases of the list, comment and update some results, and draw our conclusions.

\section{The classification}

In the SM the anomalous magnetic moment of leptons is generated through the dimensionsix effective operator

$$
\frac{1}{\Lambda^{2}} \bar{L} \sigma^{\mu \nu} e_{R} \phi F_{\mu \nu}+\text { h.c. }
$$

where $L$ is the lepton doublet, $e_{R}$ the lepton singlet, $\phi$ the Higgs doublet and $F_{\mu \nu}$ the electromagnetic field strength. This is generated at one loop, with a fermion and a gauge or Higgs boson circulating in it. In SM extensions, an analogous loop, with a new fermion or boson inside it, can give a contribution to the same operator. Here we want to classify all one-particle extensions of the SM that can contribute to the anomalous magnetic moment of leptons. As discussed before, we only look for scalars and fermions circulating in the loop. The only thing we have to require is that at any vertex Lorentz invariance, gauge invariance and renormalizability are respected. ${ }^{1}$ This gives us a finite list of new particles, that are collected in tables 1 and $2 .^{2}$ Some comments are in order.

\footnotetext{
${ }^{1} \mathrm{~A}$ non-gauge-invariant approach have been adopted in ref. [9].

${ }^{2}$ In our notation the Higgs doublet $\phi$ has hypercharge $+1 / 2$.
} 


\begin{tabular}{|c|c|c|c|c|}
\hline & $\overline{\mathrm{SU}(3)}$ & $\mathrm{SU}(2)$ & $Y$ & $Q$ \\
\hline$N_{R}$ & 1 & 1 & 0 & 0 \\
\hline$\Sigma_{R}$ & 1 & 3 & 0 & $1,0,-1$ \\
\hline$E_{4}$ & 1 & 1 & -1 & -1 \\
\hline$L_{4}$ & 1 & 2 & $-\frac{1}{2}$ & $0,-1$ \\
\hline$T$ & 1 & 3 & -1 & $0,-1,-2$ \\
\hline$D$ & 1 & 2 & $-\frac{3}{2}$ & $-2,-1$ \\
\hline
\end{tabular}

Table 1. New fermion fields with their quantum numbers.

\begin{tabular}{|ccccc|}
\hline & $\mathrm{SU}(3)$ & $\mathrm{SU}(2)$ & $Y$ & $Q$ \\
\hline$S_{1}$ & 1 & 1 & 1 & 1 \\
$S_{2}$ & 1 & 1 & 2 & 2 \\
$H_{2}$ & 1 & 2 & $\frac{1}{2}$ & 1,0 \\
$\Delta$ & 1 & 3 & 1 & $2,1,0$ \\
$T_{c}^{1 / 3}$ & $\overline{3}$ & 3 & $\frac{1}{3}$ & $\frac{4}{3}, \frac{2}{3}, \frac{1}{3}$ \\
$S_{c}^{1 / 3}$ & $\overline{3}$ & 1 & $\frac{1}{3}$ & $\frac{1}{3}$ \\
$S_{c}^{4 / 3}$ & $\overline{3}$ & 1 & $\frac{4}{3}$ & $\frac{4}{3}$ \\
$D_{c}^{7 / 6}$ & 3 & 2 & $\frac{7}{6}$ & $\frac{5}{3}, \frac{2}{3}$ \\
$D_{c}^{1 / 6}$ & 3 & 2 & $\frac{1}{6}$ & $\frac{2}{3}, \frac{1}{3}$ \\
\hline
\end{tabular}

Table 2. New scalar fields with their quantum numbers.

\subsection{Fermions}

As for new fermions, we have obtained them by looking for fermions circulating in the loop together with a Higgs boson. If on the external legs there are the lepton doublets, one gets new fermions which are colour singlets, $\mathrm{SU}(2)$ singlets or triplets, with 0 or 1 hypercharge. On the other hand, if on the external legs there are lepton singlets, one gets new fermions which are colourless, doublets under $\mathrm{SU}(2)$, with hypercharge either $-1 / 2$ or $-3 / 2$.

In principle one could think of looking for new fermions circulating in the loop together with gauge bosons. For example, if the circulating gauge bosons are the $\mathrm{SU}(2)$ ones, a new field transforming in the 4 of SU(2) would be obtained. However such kind of interaction is non-renormalizable, since it is given by the operator

$$
\bar{\psi} \sigma_{\mu \nu} \tau^{A} \chi W_{A}^{\mu \nu}
$$

where $\chi$ is the new field and $\psi$ a SM fermion. Therefore, we will not consider new fields obtained in this way, since they correspond to non-minimal extensions of the SM.

All the fermions we consider, except for the neutral ones, have to be Dirac fermions. This guarantees on the one side the presence of a Dirac mass, independent from the electroweak (EW) symmetry breaking, that can therefore assume any value and, on the other hand, that our extension of the SM is anomaly-free. Moreover, in the list of table 1, we 
recognise some well-known field: $N_{R}$ is the right-handed neutrino, which can give mass to a light neutrino via a type-I seesaw mechanism [10-13]; $\Sigma_{R}$ is a $\mathrm{SU}(2)$ triplet that can realize the type-III seesaw [14]; $L_{4}$ and $E_{4}$ are copies of the lepton doublets and singlets, but vector-like; $T$ is a triplet, whose phenomenology have been studied in ref. [15]; $D$ is a doublet recently discussed in ref. [16]. Actually these are the only fermion fields which mix with leptons at tree level respecting the SM gauge invariance; their effect on EW observable have been studied in refs. [17, 18].

\subsection{Scalars}

In the case of new scalars, more possibilities arise, depending on the fields on the external legs and inside the loop. If only lepton doublets are involved, one obtain colourless scalars with hypercharge 1 , singlets or triplets of SU(2). If only lepton singlets are there, the scalar must be colourless, $\mathrm{SU}(2)$ singlet, with hypercharge 2 . If both lepton singlets and doublets are considered, the only possibility is a replica of the Higgs doublet, with quantum numbers $(1,2,1 / 2)$.

On the other hand, if quarks circulate in the loop, the new scalars must be leptoquarks, i.e. coupling to both quarks and leptons. When there are lepton doublets on the external legs and a quark doublet in the loop, the new scalar transforms in the $\overline{3}$ of SU(3), can be either a singlet or a triplet of $\mathrm{SU}(2)$ and has hypercharge $1 / 3$. This singlet is obtained also with only singlets around, together with a similar one with hypercharge $4 / 3$. On the contrary, with doublets on the external legs and quark singlets inside the loop one obtain new scalars transforming as $\mathrm{SU}(3)$ triplets, $\mathrm{SU}(2)$ doublets and with hypercharge $1 / 6$ or $7 / 6$. This last is obtained also with leptons singlets in the external legs and a quark doublet inside. All these leptoquarks have been already classified and considered to solve the $(g-2)_{\mu}$ problem in ref. [19].

\section{The contribution of the colourless $\mathrm{SU}(2)$ doublet with hypercharge $-3 / 2$}

In this section we calculate the contribution to the muon anomalous magnetic moment given by extending the SM with a colourless $\mathrm{SU}(2)$ fermion doublet, with hypercharge $-3 / 2$, named $D$. This field is composed by two charged particles, $\chi$ and $\Psi$, with charges equal to -1 and -2 , respectively:

$$
D=\left(\begin{array}{l}
\chi \\
\Psi
\end{array}\right)
$$

The SM Lagrangian has to be enlarged to include kinetic, mass and interaction terms for $D$ :

$$
\mathcal{L}=\mathcal{L}_{\mathrm{SM}}+\bar{D}\left(\not D-M_{D}\right) D-\left(\lambda_{D} \bar{D}_{L} \phi^{c} \ell_{R}+\text { h.c. }\right)
$$

where $\lambda_{D}=\left(\lambda_{D e}, \lambda_{D \mu}, \lambda_{D \tau}\right)$ are the Yukawa couplings between $D$ and the SM leptons, $\phi^{c}=i \sigma_{2} \phi^{*}, M_{D}$ is the Dirac mass of the new field and $\mathcal{L}_{\mathrm{SM}}$ is the SM Lagrangian. 

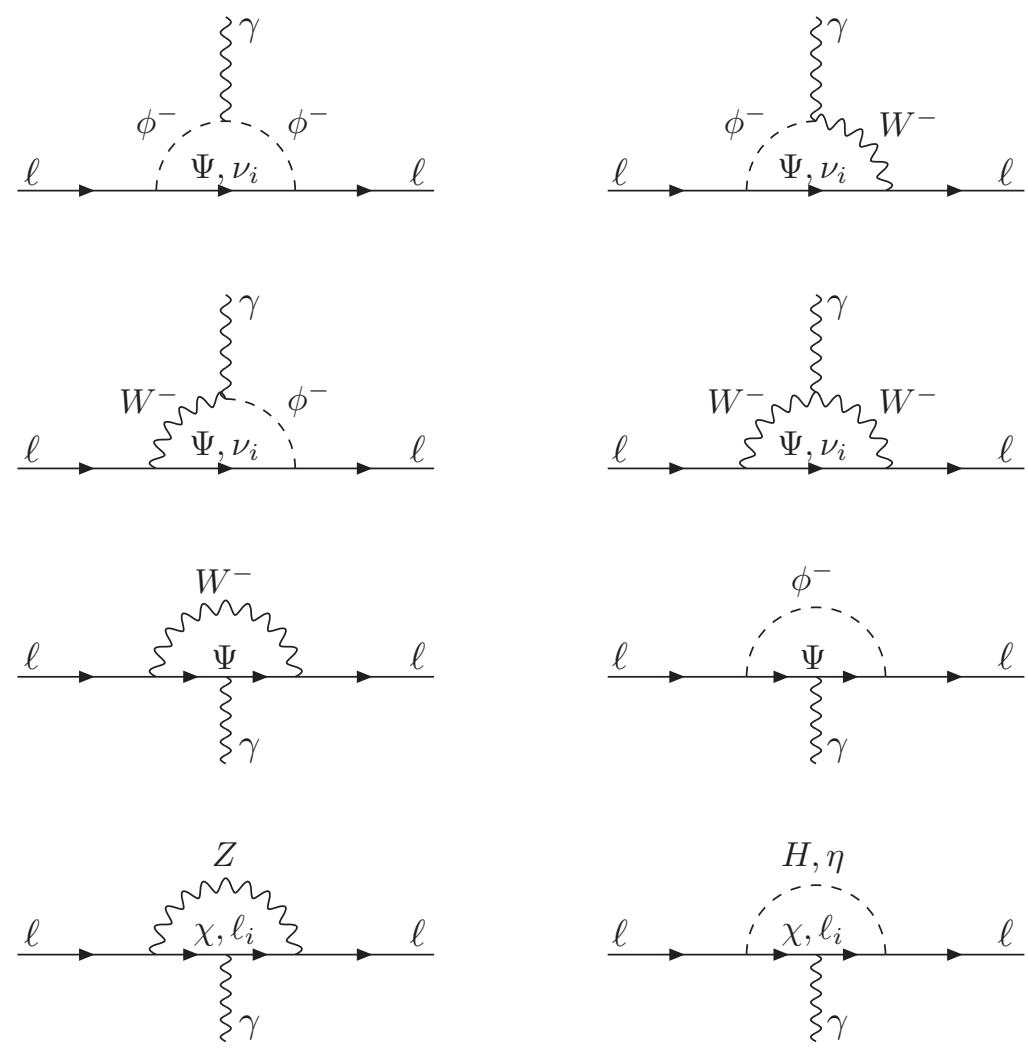

Figure 1. Diagrams contributing to the anomalous magnetic moment of the lepton $\ell$ (in the Feynman gauge). $\phi^{ \pm}$and $\eta$ are the three Goldstone bosons associated with the $W^{ \pm}$and $Z$ bosons, while $H$ stands for the physical Higgs boson.

Due to the Yukawa interaction, the singly charged component of $D, \chi$, mixes with the SM charged leptons. In the interaction basis their mass matrix is indeed given by

$$
-\left(\begin{array}{ll}
\bar{\ell}_{L} & \bar{\chi}_{L}
\end{array}\right)\left(\begin{array}{cc}
\frac{y v}{\sqrt{2}} & 0 \\
\frac{\lambda_{D} v}{\sqrt{2}} & M_{D}
\end{array}\right)\left(\begin{array}{c}
\ell_{R} \\
\chi_{R}
\end{array}\right)+\text { h.c. }
$$

where $\ell=(e \mu \tau)^{T}, y$ is the diagonal matrix of the SM leptonic Yukawa couplings and $v=246 \mathrm{GeV}$. After the diagonalisation of the mass matrix and the consequent fields redefinition, the interaction Lagrangian gets modified and the novel contribution to the anomalous magnetic moment of the muon can be calculated. We refer to the appendix for the details on the diagonalization procedure and the couplings in the mass basis.

In order to calculate the contribution of the doublet $D$ to the $(g-2)_{\mu}$, the diagrams in figure 1 have to be calculated. At $\mathcal{O}\left(\frac{v^{2} \lambda_{D} \lambda_{D}^{\dagger}}{M_{D}^{2}}\right)$, we obtain:

$$
\begin{aligned}
a_{\mu}^{\mathrm{SM}+D}=\frac{m_{\mu}^{2} G_{F}}{24 \sqrt{2} \pi^{2}}\{ & \left(3-4 \cos ^{2} \theta_{W}\right)^{2}+5+\frac{v^{2}\left|\lambda_{D \mu}\right|^{2}}{M_{D}^{2}}\left[-\frac{11}{4}-4 \cos ^{2} \theta_{W}+\right. \\
& \left.\left.+F_{\mathrm{NC}}\left(\frac{M_{D}^{2}}{M_{Z}^{2}}\right)+F_{\mathrm{h}}\left(\frac{M_{D}^{2}}{M_{H}^{2}}\right)+F_{\mathrm{CC}}\left(\frac{M_{D}^{2}}{M_{W}^{2}}\right)\right]\right\},
\end{aligned}
$$


where

$$
\begin{aligned}
F_{\mathrm{NC}}(k) & =\frac{16+14 k-63 k^{2}+38 k^{3}-5 k^{5}-6\left(2-13 k+8 k^{2}\right) \log k}{4(k-1)^{4}}, \\
F_{\mathrm{h}}(k) & =\frac{7 k^{4}-36 k^{3}+45 k^{2}-16 k+6 k(3 k-2) \log k}{4(k-1)^{4}}, \\
F_{\mathrm{CC}}(k) & =\frac{46-79 k+42 k^{2}-13 k^{3}+4 k^{4}+6\left(-4+24 k-20 k^{2}+3 k^{3}\right) \log k}{2(k-1)^{4}} .
\end{aligned}
$$

We recognize, in the $\lambda_{D}$-independent term, the well-known SM electroweak contribution [20-24]. Other terms are the contribution of the new doublet which depends on $D$ mass and couplings. The terms in the square brackets are a function of $M_{D}$, which is negative in the mass interval allowed, i.e. for $M_{D}>100.8 \mathrm{GeV}$ (LEP bounds [25]). Therefore, the addition of the doublet $D$ to the SM field content cannot explain the anomaly in anomalous magnetic moment of the muon.

Even if this model is not good to solve the discrepancy of the $(g-2)_{\mu}$, we can estimate the maximum value of this negative contribution. The combination $\frac{v^{2}\left|\lambda_{D \mu}\right|^{2}}{M_{D}^{2}}$ is indeed constrained by EW fits $[17,18]$ to be

$$
\frac{v\left|\lambda_{D \mu}\right|}{\sqrt{2} M_{D}} \leq 0.028
$$

while the maximum value of the modulus of the square bracket is obtained for the minimum allowed value for the $D$ mass, $100.8 \mathrm{GeV}$, and it corresponds to 91.8. By maximizing independently these two quantities we have an estimation of the maximum correction that the presence of such a doublet can induce in the anomalous magnetic moment of the muon:

$$
\left|a_{\mu}^{D}\right| \leq 5.5 \times 10^{-11}
$$

This value is smaller than the theoretical and the experimental errors (and thus it will not be relevant anyway in the near future).

\section{Other cases}

\subsection{Fermions}

$\mathbf{N}_{\mathbf{R}} \sim(\mathbf{1}, \mathbf{1}, \mathbf{0}) \& \boldsymbol{\Sigma}_{\mathbf{R}} \sim(\mathbf{1}, \mathbf{3}, \mathbf{0})$. The contribution to $a_{\mu}$ of these new particles has been calculated in refs. [26, 27]. Despite some minor differences in their results, the outcome is the same: their contribution is always negative and, as such, can not generate $\Delta a_{\mu}$. 
$\mathbf{E}_{4} \sim(1,1,-1) \& \mathbf{L}_{4} \sim(1,2,-1 / 2)$. The calculation in these cases has been presented in ref. [27]. ${ }^{3}$ In the $L_{4}$ case, the contribution to $a_{\mu}$ is positive, at least for masses above $119.3 \mathrm{GeV}$. This is the only case where the addition of a single fermion gives a positive contribution. However, as before, there are bounds on the combination $\frac{v^{2}\left|\lambda_{L \mu}\right|^{2}}{M_{L}^{2}}$ coming from EW fits [17, 18]:

$$
\frac{v\left|\lambda_{L \mu}\right|}{\sqrt{2} M_{D}} \leq 0.048
$$

This implies the following upper bound on the contribution to $a_{\mu}$

$$
a_{\mu}^{L_{4}}<6.8 \cdot 10^{-12}
$$

which turns out to be much smaller than what needed to solve the discrepancy between the theoretical and experimental value of $a_{\mu}{ }^{4}$

$\mathbf{T} \sim(\mathbf{1}, \mathbf{3}, \mathbf{- 1}) . \quad$ As for the contribution of the SU(2) triplet with hypercharge -1 , it has been calculated in ref. [27]: also in this case the contribution is negative, independently of the mass of the new particle, and cannot therefore explain the discrepancy.

\subsection{Scalars}

$\mathrm{S}_{1} \sim(\mathbf{1}, \mathbf{1}, \mathbf{1})$. The contribution to $a_{\mu}$ in this case has been calculated in ref. [31] and can also be derived from the results obtained more recently in ref. [28]. It turns out to be always negative and, as such, cannot solve the $a_{\mu}$ problem.

$\mathrm{S}_{2} \sim(\mathbf{1}, \mathbf{1}, \mathbf{2})$. In this case the contribution is given by two diagrams, one with the photon attached to the fermion and the other to the scalar. Also in this case it has been calculated long ago [32] and it can also be derived from the results of ref. [28]. The result is again negative, so that the addition of $S_{2}$ is not useful to explain the $a_{\mu}$ discrepancy.

$\mathbf{H}_{\mathbf{2}} \sim(\mathbf{1}, \mathbf{2}, \mathbf{- 1 / 2})$. In the case of adding a replica of the Higgs doublet, the issue is a bit more subtle, since the neutral component, which couples to the muons, can adquire a vacuum expectation value (vev).

\footnotetext{
${ }^{3}$ With respect to their results, we get a discrepancy in the constant factor in both cases, while loop functions and $\theta$-dependent terms are the same:

$a_{\mu}^{E_{4}}=\frac{G_{F} m_{\mu}^{2}}{16 \sqrt{2} \pi^{2}} \frac{v^{2}\left|\lambda_{E \mu}\right|^{2}}{M_{E}^{2}}\left[-\frac{8}{3} \cos ^{2} \theta_{w}+\frac{5}{6}+F_{F F V}\left(\frac{M_{L}^{2}}{M_{Z}^{2}}\right)+H_{F F S}\left(\frac{M_{L}^{2}}{M_{H}^{2}}\right)\right]$

$a_{\mu}^{L_{4}}=\frac{G_{F} m_{\mu}^{2}}{16 \sqrt{2} \pi^{2}} \frac{v^{2}\left|\lambda_{L \mu}\right|^{2}}{M_{L}^{2}}\left[\frac{8}{3} \cos ^{2} \theta_{w}+\frac{5}{6}+F_{F F V}\left(\frac{M_{L}^{2}}{M_{Z}^{2}}\right)+H_{F F S}\left(\frac{M_{L}^{2}}{M_{H}^{2}}\right)+2 F_{V V F}\left(\frac{M_{L}^{2}}{M_{W}^{2}}\right)+2 G_{V V F}\left(\frac{M_{L}^{2}}{M_{W}^{2}}\right)\right]$.

We have adopted the notation of ref. [27] for the loop functions. We have also cross-checked our results with ref. [28] and concluded that the discrepancy could be due to a difference in the result of the calculation of the $Z$-diagram. According to our results, the contribution of $E_{4}$ is always negative, while the contribution of $L_{4}$ is positive for masses above $119 \mathrm{GeV}$. Anyway, this difference does not modify the substance of the conclusion.

${ }^{4}$ On the contrary, notice that, as it has been shown in refs. [29, 30], the simultaneous addition of $E_{4}$ and $L_{4}$ can solve the muon $(g-2)$ puzzle.
} 
In ref. [27] they assume that the new scalar does not develope any vev contributing to the fermion masses. In this case they show that in principle there could be a positive contribution to $a_{\mu}$ able to explain the current discrepancy; however, this is now excluded by bounds coming from four-fermion interactions and searches for neutralino resonances already at LEP.

On the other hand the situation is even more involved in the case the scalar takes $v e v$, since a choice can be done regarding which of the two scalars contribute to various fermion masses. In ref. [33] they consider four two-Higgs-doublet models and conclude that in one of them $\Delta a_{\mu}$ can be obtained, thanks to the enhanced two-loops contribution of the pseudoscalar Higgs. In order for this to be realized, all the scalars must be lighter than $\sim 200 \mathrm{GeV}$, and therefore this option is testable at the LHC. For details, we remand to the mentioned paper.

$\Delta \sim(\mathbf{1}, \mathbf{3}, \mathbf{0}) . \quad$ In this case only the two charged components of the triplet contribute to $a_{\mu},{ }^{5}$ and the final result is essentially the sum of the results previously mentioned for the singly- and doubly-charged scalar. Therefore it is negative and this minimal extension of the SM can not explain the experimental result.

Leptoquarks. The possibility of explaining the $a_{\mu}$ discrepancy by adding a single leptoquark (LQ) has been discussed with great details in ref. [19], where they have concluded that this is indeed possible. In fact in this case, if the LQ has both left-handed and righthanded couplings to the muon, the contribution to $a_{\mu}$ is enhanced by the mass of the quark circulating in the loop, which must be of up-type. From our list of five leptoquarks, it can be shown that only $S_{c}^{1 / 3}$ and $D_{c}^{7 / 6}$ respect this condition and, therefore, they are good candidates to solve the $(g-2)_{\mu}$ puzzle. $^{6}$

In order to have an enhancement, either the top or the charm have to circulate in the loop. The contribution to $a_{\mu}$ will be proportional to $\frac{\lambda_{L}^{L Q \mu t(c)} \lambda_{R}^{L Q \mu t(c)}}{M_{L Q}^{2}}$ when the top (charm) is considered. Then a relevant question arises, i.e. which are the bounds on these quantities.

Let's start with the top. So far, there are no bounds coming from colliders on a leptoquark decaying into top and muon. However, in the case of $S_{c}^{1 / 3}$, bounds come from the decay into $b \nu$, giving $M_{S_{c}^{1 / 3}}>620 \mathrm{GeV}$ [36]. Even if these recent limits are stronger then the ones considered in ref. [19], they are not strong enough to rule out this possibility as an explanation of the $a_{\mu}$ anomaly. Indeed, the couplings remains perturbative $(\lambda<1(4 \pi))$ for masses smaller than 40 (500) TeV.

An example of LQ decaying in this way is the $\tilde{b}_{R}$ of the supersymmetric model of ref. [37]. Since in this model the coupling is fixed to be the Yukawa coupling, if we want to explain the $a_{\mu}$ discrepancy we obtain a prediction for the sbottom mass, which has to be around $500 \mathrm{GeV}$. This seems to be excluded from the quoted mass limit which, however, is obtained assuming a unit branching ratio. Actually, if the branching fraction is reduced

\footnotetext{
${ }^{5}$ Indeed the neutral component only couples to neutrinos [32, 34].

${ }^{6}$ These scalar LQs have been recently considered in ref. [35]. Among other things, they have shown that $D_{c}^{7 / 6}$ does not give rise to dangerous proton decay.
} 
to $60 \%$, the bound is reduced to $520 \mathrm{GeV}$ [36]. Therefore the $\tilde{b}_{R}$ of this model could still be the responsible for the disagreement between the measured and predicted values of the muon anomalous magnetic moment. This enforces the need of searches for final states with tops and muons.

On the other hand, if the scalar LQ couples to muon and charm, the stringent bounds coming from LHC searches with muons and jets in the final state apply and we have $M_{L Q}>1070 \mathrm{GeV}$ (if $\mathrm{Br}=1$ ) [38]. However, even if with this strong bound on the LQ mass larger couplings are needed, they are still perturbative, so that also a charm circulating in the loop can give a sizeable contribution to $a_{\mu}$. Indeed, in this case, the requirement of perturbativity for the LQ coupling gives the following constraint on the LQ mass: $M_{L Q}<$ $4(60) \mathrm{TeV}$ (for $\lambda<1(4 \pi)$ ), making the search at the LHC even more intriguing.

\section{Conclusions}

In this work we have completed the analysis of the contributions to the muon anomalous magnetic moment for all the one-particle extensions of the SM (scalars and fermions). In particular we have performed the calculation in the case of a colourless fermion, SU(2) doublet with hypercharge $-3 / 2$, which was absent in the literature, obtaining a negative contribution to $a_{\mu}$. From the analysis performed we can therefore conclude that the addition of a single fermion to the SM cannot explain the measuread discrepancy. On the other hand, the addition of a single scalar could be the responsible of the discrepancy, if the new scalar is a second Higgs doublet or one of the two scalar leptoquarks $S_{c}^{1 / 3}$ or $D_{c}^{7 / 6}$.

If the solution of the muon anomalous magnetic moment puzzle comes from a single new particle, this could be tested in the next run of the LHC. In the case of a second Higgs doublet, the corresponding particles should be lighter than $200 \mathrm{GeV}$ and therefore one expects that the available parameter space will be covered by the next LHC searches. On the other hand, if $\Delta a_{\mu}$ is generated by a leptoquark, mass bounds coming from the perturbativity requirement are not very stringent and in principle it could lie beyond the LHC reach. However, we have shown a particular model where this can happen and the LQ mass should be just behind the corner. Since leptoquarks have peculiar signatures at the LHC, we suggest to enforce these searches and, in particular, to look for leptoquarks decaying into top and muons, a channel not yet considered at the LHC.

\section{Acknowledgments}

We would like to thank Michele Frigerio for early discussions about this project and Lorenzo Calibbi for comments on the first version of this manuscript. This research was partially supported by the Marie Curie CIG program, project number PCIG13-GA-2013-618439, and by the Swiss National Science Foundation (SNF) under contract 200020-146644. 


\section{A Lagrangian in the mass basis}

In the interaction basis where the Lagrangian of eq. (3.2) is defined, the mass term for the singly charged leptons is given by

$$
-\left(\begin{array}{ll}
\bar{\ell}_{L} & \bar{\chi}_{L}
\end{array}\right)\left(\begin{array}{cc}
\frac{y v}{\sqrt{2}} & 0 \\
\frac{\lambda_{D} v}{\sqrt{2}} & M_{D}
\end{array}\right)\left(\begin{array}{c}
\ell_{R} \\
\chi_{R}
\end{array}\right)+\text { h.c. }
$$

The mass matrix is diagonalized with a bi-unitary transformation:

$$
\left(\begin{array}{c}
\ell_{L, R} \\
\chi_{L, R}
\end{array}\right)=V_{L, R}\left(\begin{array}{c}
\ell_{L, R}^{m} \\
\chi_{L, R}^{m}
\end{array}\right)
$$

where the superscript $m$ indicates the mass basis, and the unitary matrices performing the rotation are given by:

$$
V_{L}=\left(\begin{array}{cc}
\mathbb{1} & \frac{y \lambda_{D}^{\dagger} v^{2}}{2 M_{D}^{2}} \\
-\frac{\lambda_{D} y v^{2}}{2 M_{D}^{2}} & 1
\end{array}\right) \quad \text { and } \quad V_{R}=\left(\begin{array}{cc}
\mathbb{1}-\frac{\lambda_{D}^{\dagger} \lambda_{D} v^{2}}{4 M_{D}^{2}} & \frac{\lambda_{D}^{\dagger} v}{\sqrt{2} M_{D}} \\
-\frac{\lambda_{D} v}{\sqrt{2} M_{D}} & 1-\frac{\lambda_{D} \lambda_{D}^{\dagger} v^{2}}{4 M_{D}^{2}}
\end{array}\right)
$$

In the mass basis, the Lagrangian turns out to be:

$$
\begin{aligned}
\mathcal{L}_{\mathrm{em}} & =-e\left(\bar{l}^{m} \gamma^{\mu} l^{m}+\bar{\chi}^{m} \gamma^{\mu} \chi^{m}+2 \bar{\Psi} \gamma^{\mu} \Psi\right) A_{\mu} \\
\mathcal{L}_{\mathrm{kin}} & =i\left(\bar{l}^{m} \not l^{m}+\bar{\chi}^{m} \not \partial \chi^{m}+\bar{\Psi} \not \partial \Psi\right) \\
\mathcal{L}_{\mathrm{NC}}^{\nu \Psi} & =\frac{g}{2 \cos \theta_{w}}\left(\bar{\nu} \gamma^{\mu} P_{L} \nu+\bar{\Psi} \gamma^{\mu}\left(-1+4 \sin ^{2} \theta_{w}\right) \Psi\right) Z_{\mu} \\
\mathcal{L}_{\mathrm{NC}}^{l \chi} & =\left(\bar{l}^{m} \bar{\chi}^{m}\right) \gamma^{\mu}\left(g_{R}^{Z} P_{R}+g_{L}^{Z} P_{L}\right)\left(\begin{array}{c}
l^{m} \\
\chi^{m}
\end{array}\right) Z_{\mu} \\
\mathcal{L}_{\mathrm{CC}}^{\Psi} & =\bar{\Psi} \gamma^{\mu} W_{\mu}^{-} g_{\Psi l}^{W} l^{m}+\bar{\Psi} \gamma^{\mu} W_{\mu}^{-} g_{\Psi \chi}^{W} \chi^{m}+h . c . \\
\mathcal{L}_{\mathrm{CC}}^{\nu} & =\bar{\nu} \gamma^{\mu} W_{\mu}^{+} g_{\nu l}^{W} l^{m}+\bar{\nu} \gamma^{\mu} W_{\mu}^{+} g_{\nu \chi}^{W} \chi^{m}+h . c . \\
\mathcal{L}_{h} & =-\left(\bar{l}^{m} \bar{\chi}^{m}\right)\left(g_{R}^{h} P_{R}+g_{L}^{h} P_{L}\right)\left(\begin{array}{c}
l^{m} \\
\chi^{m}
\end{array}\right) h \\
\mathcal{L}_{\phi} & =\bar{\Psi} g_{\Psi l}^{\phi} l^{m} \phi^{-}+\bar{\Psi} g_{\Psi \chi}^{\phi} \chi^{m} \phi^{-}-\bar{l}^{m} g_{l \nu}^{\phi} \phi^{-} \nu-\bar{\chi}^{m} g_{\chi \nu}^{\phi} \nu \phi^{-}+h . c . \\
\mathcal{L}_{\eta} & =-\left(\bar{l}^{m} \bar{\chi}^{m}\right)\left(g_{R}^{\eta} P_{R}+g_{L}^{\eta} P_{L}\right)\left(\begin{array}{l}
l^{m} \\
\chi^{m}
\end{array}\right) \eta
\end{aligned}
$$


where:

$$
\begin{aligned}
& g_{R}^{Z}=\left(\begin{array}{ll}
g_{R_{l l}}^{Z} & g_{R_{l \chi}}^{Z} \\
g_{R_{\chi l}}^{Z} & g_{R_{\chi \chi}}^{Z}
\end{array}\right)=\frac{g}{\cos \theta_{w}}\left(\begin{array}{cc}
\left(1-\cos ^{2} \theta_{w}\right) \mathbb{1}+\frac{v^{2} \lambda_{D}^{\dagger} \lambda_{D}}{4 M_{D}^{2}} & -\frac{v \lambda_{D}^{\dagger}}{2 \sqrt{2} M_{D}} \\
-\frac{v \lambda_{D}}{2 \sqrt{2} M_{D}} & \frac{3}{2}-\cos ^{2} \theta_{w}-\frac{v^{2} \lambda_{D} \lambda_{D}^{\dagger}}{4 M_{D}^{2}}
\end{array}\right) \\
& g_{L}^{Z}=\left(\begin{array}{ll}
g_{L_{l l}}^{Z} & g_{L_{l \chi}}^{Z} \\
g_{L_{\chi l}}^{Z} & g_{L_{\chi \chi}}^{Z}
\end{array}\right)=\frac{g}{\cos \theta_{w}}\left(\begin{array}{cc}
\mathbb{1}\left(\frac{1}{2}-\cos ^{2} \theta_{w}\right) & -\frac{v^{2} y \lambda_{D}^{\dagger}}{2 M_{D}^{2}} \\
-\frac{v^{2} \lambda_{D} y}{2 M_{D}^{2}} & \frac{3}{2}-\cos ^{2} \theta_{w}
\end{array}\right) \\
& g_{R}^{h}=\left(\begin{array}{ll}
g_{R_{l l}}^{h} & g_{R_{l \chi}}^{h} \\
g_{R_{\chi l}}^{h} & g_{R_{\chi \chi}}^{h}
\end{array}\right)=\frac{1}{\sqrt{2}}\left(\begin{array}{cc}
y-\frac{3 y \lambda_{D}^{\dagger} \lambda_{D} v^{2}}{4 M_{D}^{2}} & \frac{y \lambda_{D}^{\dagger} v}{\sqrt{2} M_{D}} \\
\lambda_{D}+\frac{v^{2} \lambda_{D} y^{2}}{2 M_{D}^{2}}-\frac{v^{2} \lambda_{D} \lambda_{D}^{\dagger} \lambda_{D}}{4 M_{D}^{2}} & \frac{\lambda_{D} \lambda_{D}^{\dagger} v}{2 M_{D}}
\end{array}\right) \\
& g_{L}^{h}=\left(\begin{array}{ll}
g_{L_{l l}}^{h} & g_{L_{l \chi}}^{h} \\
g_{L_{\chi l}}^{h} & g_{L_{\chi \chi}}^{h}
\end{array}\right)=\frac{1}{\sqrt{2}}\left(\begin{array}{cc}
y-\frac{3 \lambda_{D}^{\dagger} \lambda_{D} y v^{2}}{4 M_{D}^{2}} & \lambda_{D}^{\dagger}+\frac{v^{2} y^{2} \lambda_{D}^{\dagger}}{2 M_{D}^{2}}-\frac{v^{2} \lambda_{D}^{\dagger} \lambda_{D} \lambda_{D}^{\dagger}}{4 M_{D}^{2}} \\
\frac{\lambda_{D} y v}{\sqrt{2} M_{D}} & \frac{\lambda_{D} \lambda_{D}^{\dagger} v}{2 M_{D}}
\end{array}\right) \\
& g_{R}^{\eta}=\left(\begin{array}{ll}
g_{R_{l l}}^{\eta} & g_{R_{l \chi}}^{\eta} \\
g_{R_{\chi l}}^{\eta} & g_{R_{\chi \chi}}^{\eta}
\end{array}\right)=\frac{i}{\sqrt{2}}\left(\begin{array}{cc}
\frac{y \lambda_{D}^{\dagger} \lambda_{D} v^{2}}{4 M_{D}^{2}}+y & \frac{y \lambda_{D}^{\dagger} v}{\sqrt{2} M_{D}} \\
-\lambda_{D}+\frac{\lambda_{D} y^{2} v^{2}}{2 M_{D}^{2}}+\frac{v^{2} \lambda_{D} \lambda_{D}^{\dagger} \lambda_{D}}{4 M_{D}^{2}}-\frac{\lambda_{D} \lambda_{D}^{\dagger} v}{\sqrt{2} M_{D}}
\end{array}\right) \\
& g_{L}^{\eta}=\left(\begin{array}{ll}
g_{L_{l l}}^{\eta} & g_{L_{l \chi}}^{\eta} \\
g_{L_{\chi l}}^{\eta} & g_{L_{\chi \chi}}^{\eta}
\end{array}\right)=\frac{i}{\sqrt{2}}\left(\begin{array}{cc}
-\frac{\lambda_{D}^{\dagger} \lambda_{D} y v^{2}}{4 M_{D}^{2}}-y+\lambda_{D}^{\dagger}-\frac{y^{2} v^{2} \lambda_{D}^{\dagger}}{2 M_{D}^{2}}-\frac{v^{2} \lambda_{D}^{\dagger} \lambda_{D} \lambda_{D}^{\dagger}}{4 M_{D}^{2}} \\
-\frac{\lambda_{D} y v}{\sqrt{2} M_{D}} & \frac{\lambda_{D} \lambda_{D}^{\dagger} v}{\sqrt{2} M_{D}}
\end{array}\right) \\
& \left\{\begin{array} { l } 
{ g _ { \nu \chi } ^ { W } = \frac { g } { \sqrt { 2 } } \frac { v ^ { 2 } y \lambda _ { D } ^ { \dagger } } { 2 M _ { D } ^ { 2 } } P _ { L } } \\
{ g _ { \nu l } ^ { W } = \frac { g } { \sqrt { 2 } } \mathbb { 1 } P _ { L } }
\end{array} \quad \left\{\begin{array}{l}
g_{\Psi l}^{W}=\frac{g}{\sqrt{2}}\left[-\frac{v^{2} \lambda_{D} y}{2 M_{D}^{2}} P_{L}-\frac{v \lambda_{D}}{\sqrt{2} M_{D}} P_{R}\right] \\
g_{\Psi \chi}^{W}=\frac{g}{\sqrt{2}}\left[P_{L}+\left(1-\frac{v^{2} \lambda_{D} \lambda_{D}^{\dagger}}{4 M_{D}^{2}}\right) P_{R}\right]
\end{array}\right.\right. \\
& \left\{\begin{array} { l } 
{ g _ { \Psi l } ^ { \phi } = \lambda _ { D } ( \mathbb { 1 } - \frac { v ^ { 2 } \lambda _ { D } ^ { \dagger } \lambda _ { D } } { 4 M _ { D } ^ { 2 } } ) P _ { R } } \\
{ g _ { \Psi \chi } ^ { \phi } = \frac { \lambda _ { D } \lambda _ { D } ^ { \dagger } v } { \sqrt { 2 } M _ { D } } P _ { R } }
\end{array} \quad \left\{\begin{array}{l}
g_{l \nu}^{\phi}=\left(y-\frac{v^{2} \lambda_{D}^{\dagger} \lambda_{D} y}{4 M_{D}^{2}}\right) P_{L} \\
g_{\chi \nu}^{\phi}=\frac{\lambda_{D} v y}{\sqrt{2} M_{D}} P_{L}
\end{array}\right.\right.
\end{aligned}
$$

Computing the diagrams in figure 1 we find the following results:

$$
\begin{aligned}
a_{\mu}^{Z \chi} & =\frac{G_{F} m_{\mu}^{2}}{24 \sqrt{2} \pi^{2}} \frac{v^{2}\left|\lambda_{D \mu}\right|^{2}}{M_{D}^{2}} \frac{8-3 k_{Z}-12 k_{Z}^{2}+7 k_{Z}^{3}+6\left(6 k_{Z}-1-4 k_{Z}^{2}\right) \log k_{Z}}{2\left(k_{Z}-1\right)^{4}} \\
a_{\mu}^{Z \mu} & =\frac{G_{F} m_{\mu}^{2}}{24 \sqrt{2} \pi^{2}}\left[\left(3-4 \cos ^{2} \theta_{w}\right)^{2}-5-\frac{2\left|\lambda_{D \mu}\right|^{2} v^{2}}{M_{D}^{2}}\left(1+2 \cos ^{2} \theta_{w}\right)\right] \\
a_{\mu}^{\eta \mu} & =\frac{11 G_{F} m_{\mu}^{2}}{24 \sqrt{2} \pi^{2}} \frac{m_{\mu}^{2}}{M_{Z}^{2}}\left(1+\frac{v^{2}\left|\lambda_{D \mu}\right|^{2}}{2 M_{D}^{2}}\right) \\
a_{\mu}^{\eta \chi} & =\frac{G_{F} m_{\mu}^{2}}{16 \sqrt{2} \pi^{2}} \frac{\left|\lambda_{D \mu}\right|^{2} v^{2}}{M_{D}^{2}} \frac{-5 k_{Z}^{4}+24 k_{Z}^{3}-39 k_{Z}^{2}+20 k_{Z}+6 k_{Z} \log \left(k_{Z}\right)}{6\left(k_{Z}-1\right)^{4}}
\end{aligned}
$$




$$
\begin{aligned}
a_{\mu}^{h \chi} & =\frac{G_{F} m_{\mu}^{2}}{16 \sqrt{2} \pi^{2}} \frac{\left|\lambda_{D \mu}\right|^{2} v^{2}}{M_{D}^{2}} \frac{7 k_{h}^{4}-36 k_{h}^{3}+45 k_{h}^{2}-16 k_{h}+6 k_{h}\left(3 k_{h}-2\right) \log \left(k_{h}\right)}{6\left(k_{h}-1\right)^{4}} \\
a_{\mu}^{h \mu} & =-\frac{7 G_{F} m_{\mu}^{2}}{24 \sqrt{2} \pi^{2}} \frac{m_{\mu}^{2}}{m_{h}^{2}}\left(1-\frac{3\left|\lambda_{D \mu}\right|^{2}}{2 M_{D}^{2}}\right) \\
a_{\mu}^{W \nu} & =\frac{7 G_{F} m_{\mu}^{2}}{24 \sqrt{2} \pi^{2}} \\
a_{\mu_{1}}^{W \Psi} & =\frac{G_{F} m_{\mu}^{2}}{8 \sqrt{2} \pi^{2}} \frac{v^{2}\left|\lambda_{D \mu}\right|^{2}}{M_{D}^{2}} \frac{11-57 k_{W}+69 k_{W}^{2}-23 k_{W}^{3}+6 k_{W}^{2}\left(-5+3 k_{W}\right) \log k_{W}}{6\left(k_{W}-1\right)^{4}} \\
a_{\mu_{2}}^{W \Psi} & =\frac{G_{F} m_{\mu}^{2}}{2 \sqrt{2} \pi^{2}} \frac{v^{2}\left|\lambda_{D \mu}\right|^{2}}{M_{D}^{2}} \frac{8-3 k_{W}-12 k_{W}^{2}+7 k_{W}^{3}-6\left(1-6 k_{W}+4 k_{W}^{2}\right) \log k_{W}}{6\left(k_{W}-1\right)^{4}} \\
a_{\mu}^{\phi \nu_{\mu}} & =-\frac{G_{F} m_{\mu}^{2}}{24 \sqrt{2}} \frac{m_{\mu}^{2}}{M_{W}^{2}}\left(1-\frac{\left|\lambda_{D \mu}\right|^{2} v^{2}}{2 M_{D}^{2}}\right) \\
a_{\mu_{1}}^{\phi \Psi} & =\frac{G_{F} m_{\mu}^{2}}{8 \sqrt{2} \pi^{2}} \frac{v^{2}\left|\lambda_{D \mu}\right|^{2}}{M_{D}^{2}} k_{W} \frac{\left(k_{W}-1\right)\left[-1+k_{W}\left(5+2 k_{W}\right)\right]-6 k_{W}^{2} \log k_{W}}{6\left(k_{W}-1\right)^{4}} \\
a_{\mu_{2}}^{\phi \Psi} & =\frac{G_{F} m_{\mu}^{2}}{4 \sqrt{2} \pi^{2}} \frac{v^{2}\left|\lambda_{D \mu}\right|^{2}}{M_{D}^{2}} k_{W} \frac{2+3 k_{W}-6 k_{W}^{2}+k_{W}^{3}+6 k_{W} \log k_{W}}{6\left(k_{W}-1\right)^{4}} \\
a_{\mu}^{W \phi} & =\frac{G_{F} m_{\mu}^{2}}{16 \sqrt{2} \pi^{2}}\left(1-\frac{v^{2}\left|\lambda_{D \mu}\right|^{2}}{4 M_{D}^{2}}\right) \\
a_{\mu}^{W \phi \Psi} & =\frac{G_{F} m_{\mu}^{2}}{16 \sqrt{2} \pi^{2}} \frac{v^{2}\left|\lambda_{D \mu}\right|^{2}}{M_{D}^{2}} \frac{-1+\left(4-3 k_{W}\right) k_{W}+2 k_{W}^{2} \log k_{W}}{2\left(k_{W}-1\right)^{3}}
\end{aligned}
$$

where $k_{Z}=M_{D}^{2} / M_{Z}^{2}, k_{W}=M_{D}^{2} / M_{W}^{2}$ and $k_{h}=M_{D}^{2} / M_{h}^{2}$. Summing all these contributions together (and twice the last two since each of them corresponds to two diagrams) we obtain the result shown in eq. (3.4).

Open Access. This article is distributed under the terms of the Creative Commons Attribution License (CC-BY 4.0), which permits any use, distribution and reproduction in any medium, provided the original author(s) and source are credited.

\section{References}

[1] Muon G-2 collaboration, G.W. Bennett et al., Final Report of the Muon E821 Anomalous Magnetic Moment Measurement at BNL, Phys. Rev. D 73 (2006) 072003 [hep-ex/0602035] [INSPIRE].

[2] F. Jegerlehner and A. Nyffeler, The Muon g-2, Phys. Rept. 477 (2009) 1 [arXiv:0902.3360] [INSPIRE].

[3] M. Davier, S. Eidelman, A. Hocker and Z. Zhang, Updated estimate of the muon magnetic moment using revised results from $e^{+} e^{-}$annihilation, Eur. Phys. J. C 31 (2003) 503 [hep-ph/0308213] [INSPIRE].

[4] M. Passera, W.J. Marciano and A. Sirlin, The Muon g-2 and the bounds on the Higgs boson mass, Phys. Rev. D 78 (2008) 013009 [arXiv:0804.1142] [INSPIRE]. 
[5] M. Passera, W.J. Marciano and A. Sirlin, The muon g-2 discrepancy: Errors or new physics?, AIP Conf. Proc. 1078 (2009) 378 [arXiv:0809.4062] [InSPIRE].

[6] M. Passera, W.J. Marciano and A. Sirlin, The Muon g-2 Discrepancy: New Physics or a Relatively Light Higgs?, Chin. Phys. C 34 (2010) 735 [arXiv:1001.4528] [INSPIRE].

[7] R.M. Carey et al., The New (g-2) Experiment: A proposal to measure the muon anomalous magnetic moment to \pm 0.14 ppm precision, FERMILAB-PROPOSAL-0989 (2009) [INSPIRE].

[8] http://j-parc.jp/researcher/Hadron/en/pac_0907/pdf/LOI_Saito.pdf.

[9] F.S. Queiroz and W. Shepherd, New Physics Contributions to the Muon Anomalous Magnetic Moment: A Numerical Code, Phys. Rev. D 89 (2014) 095024 [arXiv:1403.2309] [INSPIRE].

[10] P. Minkowski, $\mu \rightarrow$ ey at a Rate of One Out of 1-Billion Muon Decays?, Phys. Lett. B 67 (1977) 421 [inSPIRE].

[11] M. Gell-Mann, P. Ramond and R. Slansky, in Supergravity, P. van Nieuwenhuizen and D. Freedman eds., North-Holland, Amsterdam The Netherlands (1979), pg. 315.

[12] T. Yanagida, in Proceedings of the Workshop on the Unified Theory and the Baryon Number in the Universe, O. Sawada and A. Sugamoto eds., KEK Report No. 79-18, Tsukuba Japan (1979), pg. 95.

[13] R.N. Mohapatra and G. Senjanović, Neutrino Mass and Spontaneous Parity Violation, Phys. Rev. Lett. 44 (1980) 912 [InSPIRE].

[14] R. Foot, H. Lew, X.G. He and G.C. Joshi, Seesaw Neutrino Masses Induced by a Triplet of Leptons, Z. Phys. C 44 (1989) 441 [inSPIRE].

[15] A. Delgado, C. Garcia Cely, T. Han and Z. Wang, Phenomenology of a lepton triplet, Phys. Rev. D 84 (2011) 073007 [arXiv:1105.5417] [InSPIRE].

[16] T. Ma, B. Zhang and G. Cacciapaglia, Doubly Charged Lepton from an Exotic Doublet at the LHC, Phys. Rev. D 89 (2014) 093022 [arXiv: 1404.2375] [INSPIRE].

[17] F. del Aguila, J. de Blas and M. Pérez-Victoria, Effects of new leptons in Electroweak Precision Data, Phys. Rev. D 78 (2008) 013010 [arXiv: 0803.4008] [INSPIRE].

[18] J. de Blas, Electroweak limits on physics beyond the Standard Model, EPJ Web Conf. 60 (2013) 19008 [arXiv:1307.6173] [INSPIRE].

[19] D. Chakraverty, D. Choudhury and A. Datta, A Nonsupersymmetric resolution of the anomalous muon magnetic moment, Phys. Lett. B 506 (2001) 103 [hep-ph/0102180] [INSPIRE].

[20] R. Jackiw and S. Weinberg, Weak interaction corrections to the muon magnetic moment and to muonic atom energy levels, Phys. Rev. D 5 (1972) 2396 [InSPIRE].

[21] I. Bars and M. Yoshimura, Muon magnetic moment in a finite theory of weak and electromagnetic interaction, Phys. Rev. D 6 (1972) 374 [INSPIRE].

[22] G. Altarelli, N. Cabibbo and L. Maiani, The Drell-Hearn sum rule and the lepton magnetic moment in the Weinberg model of weak and electromagnetic interactions, Phys. Lett. B 40 (1972) 415 [INSPIRE].

[23] W.A. Bardeen, R. Gastmans and B.E. Lautrup, Static quantities in Weinberg's model of weak and electromagnetic interactions, Nucl. Phys. B 46 (1972) 319 [INSPIRE]. 
[24] K. Fujikawa, B.W. Lee and A.I. Sanda, Generalized Renormalizable Gauge Formulation of Spontaneously Broken Gauge Theories, Phys. Rev. D 6 (1972) 2923 [inSPIRE].

[25] Particle Data Group collaboration, K.A. Olive et al., Review of Particle Physics, Chin. Phys. C 38 (2014) 090001.

[26] C. Biggio, The Contribution of fermionic seesaws to the anomalous magnetic moment of leptons, Phys. Lett. B 668 (2008) 378 [arXiv:0806.2558] [INSPIRE].

[27] A. Freitas, J. Lykken, S. Kell and S. Westhoff, Testing the Muon g-2 Anomaly at the LHC, JHEP 05 (2014) 145 [Erratum ibid. 1409 (2014) 155] [arXiv: 1402.7065] [INSPIRE].

[28] W.-C. Chiu, C.-Q. Geng and D. Huang, Correlation Between Muon $g-2$ and $\mu \rightarrow e \gamma$, Phys. Rev. D 91 (2015) 013006 [arXiv: 1409.4198] [INSPIRE].

[29] K. Kannike, M. Raidal, D.M. Straub and A. Strumia, Anthropic solution to the magnetic muon anomaly: the charged see-saw, JHEP 02 (2012) 106 [Erratum ibid. 1210 (2012) 136] [arXiv:1111.2551] [INSPIRE].

[30] R. Dermisek and A. Raval, Explanation of the Muon g-2 Anomaly with Vectorlike Leptons and its Implications for Higgs Decays, Phys. Rev. D 88 (2013) 013017 [arXiv:1305.3522] [INSPIRE].

[31] J.A. Coarasa Perez, A. Mendez and J. Solà, Higgs triplet effects in purely leptonic processes, Phys. Lett. B 374 (1996) 131 [hep-ph/9511297] [INSPIRE].

[32] J.F. Gunion, J. Grifols, A. Mendez, B. Kayser and F.I. Olness, Higgs Bosons in Left-Right Symmetric Models, Phys. Rev. D 40 (1989) 1546 [InSPIRE].

[33] A. Broggio, E.J. Chun, M. Passera, K.M. Patel and S.K. Vempati, Limiting two-Higgs-doublet models, JHEP 11 (2014) 058 [arXiv: 1409.3199] [INSPIRE].

[34] A. Abada, C. Biggio, F. Bonnet, M.B. Gavela and T. Hambye, Low energy effects of neutrino masses, JHEP 12 (2007) 061 [arXiv:0707.4058] [INSPIRE].

[35] F.S. Queiroz, K. Sinha and A. Strumia, Leptoquarks, Dark Matter and Anomalous LHC Events, arXiv:1409.6301 [INSPIRE].

[36] ATLAS collaboration, Search for direct third-generation squark pair production in final states with missing transverse momentum and two b-jets in $\sqrt{s}=8 \mathrm{TeV}$ pp collisions with the ATLAS detector, JHEP 10 (2013) 189 [arXiv:1308.2631] [INSPIRE].

[37] F. Riva, C. Biggio and A. Pomarol, Is the $125 \mathrm{GeV}$ Higgs the superpartner of a neutrino?, JHEP 02 (2013) 081 [arXiv:1211.4526] [INSPIRE].

[38] CMS collaboration, Search for Pair-production of Second generation Leptoquarks in $8 \mathrm{TeV}$ proton-proton collisions, CMS-PAS-EXO-12-042 (2012). 